\title{
Surgical Management of Vesical Stones in Children: A Comparison Between Open Cystolithotomy, Percutaneous Cystolithotomy and Transurethral Cystolithotripsy With Holmium-YAG Laser
}

\author{
Babak Javanmard ${ }^{1}$, Morteza Fallah Karkan², Mohammad Reza Razzaghi², Saleh Ghiasy², Arash Ranjbar ${ }^{2}$, \\ Amirhossein Rahavian ${ }^{2 *}$ \\ ${ }^{1}$ Shohada-e-Tajrish Hospital, Shahid Beheshti University of Medical Sciences, Tehran, Iran \\ ${ }^{2}$ Laser Application in Medical Science Research Center, Shahid Beheshti University of Medical Sciences, Tehran, Iran
}

\author{
*Correspondence to \\ Amirhossein Rahavian, MD; \\ Urology resident, Laser \\ Application in Medical Science \\ Research Center, Shahid \\ Beheshti University of Medical \\ Sciences, Tehran, Iran. \\ Tel: +982122749221 \\ Email: \\ amirrahavian@yahoo.com
}

Published online 28 July 2018

\begin{abstract}
Introduction: Bladder stone is more common in developing countries and it is one of the rare diseases affecting children. In recent years, there is an increasing tendency in urologists to have minimal invasive approaches. The aim of this study was to compare the results of surgical bladder stone management in our single tertiary center.

Methods: From 159 children younger than 18 years with vesical stones treated during January 2000 to January 2015 in Shohada-e-Tajrish hospital, Tehran, Iran, 146 subjects entered to the study. According to type of intervention, patients stratified to three groups. First group included 80 patients that open cystolithotomy $(\mathrm{OCL})$ was done for them, second group was 39 patients who underwent percutaneous cystolithotomy (PCCL) and third group was 27 patients who treated by transurethral cystolithotripsy with holmium-YAG laser (TULL). Demographic data, hospital stay, operation time, and post operation complications were recorded and comparison were drawn between the three groups.

Results: Mean age was $8.3 \pm 5.1$ years $(8.6 \pm 5.1,6 \pm 4.2$ years for boys and girls, respectively). Mean stone size (MSS) was $2.76 \pm 1.07 \mathrm{~cm}$ and no significant differences were found between the three groups but it was larger in OCL group. Mean operative time (MOT) was $29.15 \pm 7.12$ minutes in separate; MOT in TULL was significantly higher than PCCL and OCL respectively $(36.3 \pm 5.97$, $30.54 \pm 5.27$ and $26.06 \pm 6.32$ minutes $/ P=0.000$ ). Mean hospital stay $(M H S)$ in $O C L$ group was $3.55 \pm 1$ days that was higher than PCCL and TULL groups significantly $(P=0.000)$.

Conclusion: Based on our study, holmium:YAG lithotripsy has a high success rate, and is a minimally invasive management, therefore it is a safe method for children bladder calculi treatment if proper equipment was available and done by expert hands.

Keywords: Bladder stone; Children; Open cystolithotomy; Percutaneous cystolithotomy; Transurethral cystolithotripsy; Holmium-YAG laser.
\end{abstract}

\section{Introduction}

Bladder calculi in children is a rare disease and constitutes $5 \%$ of urolithiasis in western countries ${ }^{1}$ but in developing countries and in huge areas of Iran, China, Turkey, India, and Indonesia more children are suffering from it due to high prevalence of urinary tract infection (UTI) and poor nutrition state especially protein-poor diet. ${ }^{2,3}$

Although the best option for managing these patients is surgery and open cystolithotomy (OCL) is considered as the gold standard with high stone-free rate, ${ }^{4,5}$ by means of developing in endourologic methods and endourologists experiences PCCL becomes a safe alternative for large bladder stones. It provides high stone-free rate with less scar and morbidity in comparison with open approach. ${ }^{6,7}$ Nowadays due to the development of small equipment associated with entrance of laser science to medicine, using laser for management of urolithiasis is a good choice. ${ }^{8,9}$ Likewise, because of small size of urethra in children, transurethral approach for bladder calculi lithotripsy by laser becomes popular among urologists and some studies showed its safety and efficacy, however more data are still needed. ${ }^{6,10}$

The purpose of this survey was to compare the results of surgical bladder stone management in our single tertiary center. 


\section{Methods}

Study Population

A retrospective review from April 2000 to January 2015 was carried out on 159 pediatric patients up to 18 years old and 146 children (129 boys with male/ female ratio: $7.5 / 1$ ) that had vesical stones were enrolled in the study conducted in Shohada-e-Tajrish hospital, tertiary referral center, Tehran, Iran. Thirteen patients were excluded from the study due to lack of clinical and surgical data.

These subjects were categorized into the 3 groups based on surgical management methods including percutaneous cystolithotomy (PCCL) (39), OCL (80) and transurethral cystolithotripsy with holmium laser (TULL) (27). Three of the patients had a previous history of bladder stone surgery in the center, hence two of them had history of OCL and the other one had a history of PCCL. In the second admission all of them were treated via TULL as well. The data of these patients recorded and analyzed twice in their respective groups. The mean age was $8.3 \pm 5.1$ years (mean age stratified by procedure: OCL 7.6 \pm 5.1 , PCCL 9.2 \pm 5.1 , and TULL $9 \pm 5$ years).

The diagnosis was based on plain abdominal radiography and ultrasonography. Urine culture, renal function test and coagulative status test were done for all the patients before procedure, and if needed, antibiotic therapy was carried out.

\section{Study Outcome}

Demographics data, Clinical features, intraoperative and postoperative complications were recorded. Operative times from beginning of urethrocystoscopy till catheter indwelling; and postoperative hospital stay were compared.

\section{Surgical Technique}

Single dose prophylactic intravenous antibiotics were administered before surgery. All patients underwent general anesthesia and placed in supine or lithotomy position according to the procedure. Cystourethroscopy was done for all patients to exclude urethral abnormality or to retropulsion the urethral stone into the bladder before main operation.

\section{PCCL}

In supine position after filling the bladder during urethrocystoscopy, $2 \mathrm{~cm}$ above pubic symphysis, 1 $\mathrm{cm}$ transverse incision was done and 18 gauge needle bladder was punctured then dilatation was performed and 26 Fr or 28 Fr Amplatz sheath was inserted and with 24 Fr nephroscope and LithoClast lithotripter (Swiss LithoClast) stone fragmentation was done and particles were extracted with grasper. Finally, cystostomy and urethral catheter were inserted. Postoperative, cystostomy and catheter were discharged after 1 and 2 days respectively. If there was no complication, patients were discharged after 2 or 3 days.

\section{OCL}

In supine position a suprapubic midline 3 to $4 \mathrm{~cm}$ incision was performed and after extraction of stone and bladder repairing a tube drain was inserted. As a rule, urethral catheter was removed in 5 days later but tube drain and patient was discharged after 3 days.

\section{TULL}

In lithotomy position a 6.5 Fr Semirigid Ureteroscope (Wolf Inc., Germany) was introduced to the bladder and holmium: YAG laser (Ho: YAG) (Iranian National Laser Center, Iran); via $200 \mu \mathrm{m}$ fiber was applied at power of 5 to $10 \mathrm{~W}$ with frequency of $8-10 \mathrm{~Hz}$ to vaporize the stone and at the end indwelling catheter was inserted. As a rule, patient along with the catheter was discharged 1 day after operation.

\section{Data Analysis}

Statistical Package for the Social Sciences (SPPS) (version 20) was performed using chi-square tests for qualitative data and quantitative outcomes were showed through descriptive statistics (mean \pm standard deviation) and one-way analysis of variance (ANOVA) test. The $P<0.05$ was considered as the statistical significance level.

\section{Results}

From 146 children that were enrolled in our study 33 children $(20.7 \%)$ had positive urine culture and treated pre-operation based on culture sensitivity (the dominant organism was Escherichia coli (66.6\%)). Mean age was $8.3 \pm 5.1$ years (boys $8.6 \pm 5.1$ and girls $6 \pm 4.2$ years).

Mean stone size (MSS) was $2.76 \pm 1.07 \mathrm{~cm}$ and no significant differences were found between the three groups but the MSS was larger in OCL group.

Mean operative time (MOT) was $29.15 \pm 7.12$ minutes that in separate, MOT of the TULL group was significantly higher than PCCL and OCL groups respectively $(36.3 \pm 5.97,30.54 \pm 5.27$ and $26.06 \pm 6.32 \mathrm{~min} / P=0.000)$.

Mean hospital stay (MHS) in OCL group was $3.55 \pm 1$ days that was significantly higher than PCCL and TULL groups $(P=0.000)$ and PCCL group had significant higher hospital stay than TULL group $(P=0.000)$. All the data are shown in Table 1 in details.

All the children in OCL group were discharged 3-4 days after operation if there were no complications. One patient who underwent OCL needed reoperation due to urinary leakage and perivesical collection so his hospital stay was prolonged (8 days). Five patients developed with irritative symptoms and four children encountered with surgical site infection so their hospital stay was longer. Hospital stay of the 5 children in the PCCL group was longer due to surgical site infection (2), urinary leakage (1) and irritative symptoms (2).

In the TULL group there was no major bladder trauma like bladder perforation, but 5 patients developed with hematuria and just one patient developed with gross 
Table 1. Demographic and Clinical Data

\begin{tabular}{|c|c|c|c|c|c|}
\hline Variable & All Cases $(N=146)$ & OCL $(n=80)$ & PCCL $(n=39)$ & TULL $(n=27)$ & $P$ Value \\
\hline Gender/male & 129 & 73 & 34 & 22 & \\
\hline Age $(y)$ & $8.3 \pm 5.1(0.5-17.5)$ & $7.6 \pm 5.1(0.5-17.5)$ & $9.2 \pm 5.1(1.5-17)$ & $9 \pm 5(1.5-17)$ & \\
\hline Stone size $(\mathrm{cm})$ & $2.76 \pm 1.07(1-6.2)$ & $2.89 \pm 1.17(1-6.2)$ & $2.6 \pm 1(1-4.9)$ & $2.54 \pm 0.8(1-4.5)$ & 0.618 \\
\hline MOT (min) & $29.15 \pm 7.12(15-54)$ & $26.06 \pm 6.32(15-48)$ & $30.54 \pm 5.27(22-42)$ & $36.30 \pm 5.97(28-54)$ & 0.000 \\
\hline MHS (d) & $2.85 \pm 1.23(1-8)$ & $3.55 \pm 1(2-8)$ & $2.49 \pm 0.72(2-4)$ & $1.3 \pm 0.66(1-4)$ & 0.001 \\
\hline Stone composition & $\begin{array}{l}\text { 94: Calcium-oxalate (51), } \\
\text { ammonium acid urate (28), } \\
\text { other (15) }\end{array}$ & $\begin{array}{l}\text { 47: calcium-oxalate } \\
\text {-oxalate (28), ammonium } \\
\text { acid urate (19) }\end{array}$ & $\begin{array}{l}\text { 32: calcium-oxalate -oxalate } \\
\text { (23), ammonium acid urate } \\
\text { (9) }\end{array}$ & $\mathrm{N} / \mathrm{A}$ & \\
\hline
\end{tabular}

Abbreviations: OCL, open cystolithotomy; PCCl, Percutaneous cystolithotomy; TULL, transurethral cystolithotripsy with holmium-YAG laser; MOT, mean operative time; MHS, mean hospital stay.

N/A: Not Available due to vaporization mechanism of laser.

hematuria that with bladder irrigation the problem was solved. The five children had mild urinary irritative symptoms that were controlled by anticholinergic agents and they were discharged one day later.

Three of these children ( 2 in the TULL group and 1 in the PCCL) developed bulbar urethral stricture that was managed with dilatation once and they did not come back again.

The data of 129 patients that were followed by bladder sonography two weeks after operation revealed that all of them were stone free and the others were not followed.

Four patients ( 2 boys) had history of neurogenic bladder so cystoplasty augmentation and routinely PCCL were performed for them but for 1 of them during PCCL due to the mechanical problem of LithoClast the operation was changed to OCL and this patient was stratified to OCL group.

One of the patients in the OCL group was proven neurogenic bladder that cystoplasty augmentation and OCL were done simultaneously; therefore operation time was calculated from skin incision till stone extraction.

Posterior urethral valve (PUV) was revealed for 4 boys in TULL group during operation so ablation and laser lithotripsy was done in one session. One boy was known as a case of urethral stricture due to traumatic catheterization that internal urethrotomy and laser lithotripsy were done concurrently. The operation time was arbitrated after PUV ablation or urethrotomy till indwelling catheter insertion.

Stone composition was available in 94 children that calcium oxalate (51) and ammonium acid urate (28) were dominant in order.

\section{Discussion}

Voiding dysfunctions, foreign bodies, urethral strictures, PUV and vesicoureteral reflux predispose children to vesicolithiasis. ${ }^{11}$

Lal et al reported that the incidence of vesical stone in children is slowly declining. This is predominantly due to nutritional improvement, better pre-and postnatal care and improvement of the people's awareness in the endemic areas. ${ }^{12}$

Vesical calculi could be treated by open surgery to transurethral or percutaneous suprapubic lithotripsy. ${ }^{13}$

Development in pediatric ureteroscopes and cystoscopes and presentation of Ho:YAG laser, causes a steady shift to TULL in children's bladder stone management. ${ }^{11}$

Several studies have mentioned the advantages of Ho:YAG laser lithotripsy for managing vesical lithiasis, in comparison with other choices. ${ }^{10}$

Traditionally, open surgery was the choice of treatment for large bladder stone $(>4 \mathrm{~cm})$. However, some studies revealed that the use Ho:YAG laser was successful in managing the stones with mean diameter of $5.5-6 \mathrm{~cm} \cdot{ }^{14,15}$ Several studies compared 2 types of bladder stone surgical managements in children ${ }^{7,11,16}$ but just one study (based on our search) compared 3 modalities of surgery. ${ }^{13}$ In this research we investigated three types of surgical managements with an appropriate sample size.

It was demonstrated that MOT in OCL group was significantly lower than the other 2 groups $(26.06 \pm 6.32$ minutes, $P=0.000)$, besides there was a significant difference between MHS of the TULL group and the other groups $(1.3 \pm 0.66$ days, $P=0.001)$.

Isen et al studied the treatment of vesical stones $(<2 \mathrm{~cm})$ in children ( 27 boys $3-14 \mathrm{y} / \mathrm{o}$ ) by transurethral pneumatic lithotripsy using an ureteroscope (8 Fr to $9.8 \mathrm{Fr}$ Wolf semirigid ureteroscope or 9.5 Fr Storz ureteroscope). They concluded pneumatic lithotripsy with ureteroscope is a safe and effective method for bladder stones management in children. ${ }^{17}$

Al-Marhoon et al compared PCCL (27 patients that 10 of them were treated with Ho:YAG cystolithotripsy), and OCL (53 subjects) in children with bladder lithiasis. The MSS was $3.1 \pm 1.6 \mathrm{~cm}$ in the OCL and $1.8 \pm 0.8 \mathrm{~cm}$ in the PCCL groups. The operation length was comparable between the groups and the hospital stay was shorter in the PCCL (2.6 vs 4.8 days). In the OCL group, one child had a small bowel injury, while three children had intraperitoneal bladder injury and one child had continues 
Table 2. Comparison Between other studies and Our Results

\begin{tabular}{|c|c|c|c|c|c|c|c|c|c|c|c|}
\hline \multirow{2}{*}{ Studies } & \multirow{2}{*}{$\begin{array}{l}\text { No. of } \\
\text { Cases }\end{array}$} & \multicolumn{3}{|l|}{ MSS (cm) } & \multicolumn{3}{|c|}{ MHS (d) } & \multicolumn{3}{|l|}{ MOT (min) } & \multirow{2}{*}{ Ref. } \\
\hline & & OCL & PCCL & TULL & OCL & PCCL & TULL & OCL & PCCL & TULL & \\
\hline Current study & 146 & $2.89 \pm 1.17$ & $2.6 \pm 1$ & $2.54 \pm 0.8$ & $3.55 \pm 1$ & $2.49 \pm 0.72$ & $1.3 \pm 0.66$ & $26.06 \pm 6.32$ & $30.54 \pm 5.27$ & $36.30 \pm 5.97$ & \\
\hline $\begin{array}{l}\text { Nameirakpam } \\
\text { (2016) }\end{array}$ & 85 & $\mathrm{~N} / \mathrm{A}^{\mathrm{a}}$ & $\mathrm{N} / \mathrm{A}$ & $3 \pm 1.2$ & $\mathrm{~N} / \mathrm{A}$ & $\mathrm{N} / \mathrm{A}$ & 0 & $\mathrm{~N} / \mathrm{A}$ & $\mathrm{N} / \mathrm{A}$ & $40 \pm 10$ & 20 \\
\hline Lal (2015) & 113 & 2.5 & N/A & $\mathrm{N} / \mathrm{A}$ & 4 & $\mathrm{~N} / \mathrm{A}$ & $\mathrm{N} / \mathrm{A}$ & $\mathrm{N} / \mathrm{A}$ & $\mathrm{N} / \mathrm{A}$ & $\mathrm{N} / \mathrm{A}$ & 12 \\
\hline $\begin{array}{l}\text { Aboulela } \\
(2015)\end{array}$ & 33 & $\mathrm{~N} / \mathrm{A}$ & $\mathrm{N} / \mathrm{A}$ & $2.02 \pm 0.82$ & $\mathrm{~N} / \mathrm{A}$ & $\mathrm{N} / \mathrm{A}$ & $\mathrm{N} / \mathrm{A}$ & $\mathrm{N} / \mathrm{A}$ & $\mathrm{N} / \mathrm{A}$ & $31.21 \pm 9.1$ & 11 \\
\hline $\begin{array}{l}\text { Ahmadnia } \\
\text { (2013) }\end{array}$ & 147 & $\mathrm{~N} / \mathrm{A}$ & 2.74 & $\mathrm{~N} / \mathrm{A}$ & $\mathrm{N} / \mathrm{A}$ & 1.2 & $\mathrm{~N} / \mathrm{A}$ & $\mathrm{N} / \mathrm{A}$ & 29.7 & $\mathrm{~N} / \mathrm{A}$ & 15 \\
\hline $\begin{array}{l}\text { Al-Mahroon } \\
(2009)\end{array}$ & 107 & $3.1 \pm 1.6$ & $1.8 \pm 0.8$ & $1.2 \pm 0.6$ & 2.6 & 4.8 & & $38 \pm 12$ & $46 \pm 14$ & & 13 \\
\hline $\begin{array}{l}\text { Ahmadnia } \\
\text { (2006) }\end{array}$ & 30 & $\mathrm{~N} / \mathrm{A}$ & $24.8 \pm 8.47$ & $\mathrm{~N} / \mathrm{A}$ & $\mathrm{N} / \mathrm{A}$ & $\mathrm{N} / \mathrm{A}$ & $\mathrm{N} / \mathrm{A}$ & $\mathrm{N} / \mathrm{A}$ & $23.13 \pm 8.38$ & $\mathrm{~N} / \mathrm{A}$ & 7 \\
\hline Salah (2005) & 155 & $\mathrm{~N} / \mathrm{A}$ & 2.3 & $\mathrm{~N} / \mathrm{A}$ & $\mathrm{N} / \mathrm{A}$ & 2.7 & $\mathrm{~N} / \mathrm{A}$ & $\mathrm{N} / \mathrm{A}$ & 20 & $\mathrm{~N} / \mathrm{A}$ & 21 \\
\hline $\begin{array}{l}\text { Ramakrishnan } \\
(2005)\end{array}$ & 23 & $\mathrm{~N} / \mathrm{A}$ & $\mathrm{N} / \mathrm{A}$ & 27 & $\mathrm{~N} / \mathrm{A}$ & N/A & 2.2 & $\mathrm{~N} / \mathrm{A}$ & $\mathrm{N} / \mathrm{E}$ & 38 & 19 \\
\hline
\end{tabular}

Abbreviation: OCL, open cystolithotomy; PCCl, Percutaneous cystolithotomy; TULL, transurethral cystolithotripsy with holmium-YAG laser; MOT, mean operative time; MHS, mean hospital stay; MSS, Mean stone size.

a Not Assess.

urinary leakage from the site of operation in the PCCL group. They reported endourological management caused a shorter hospital admission but the OCL was safer. Also they recommended using fine flexible ureteroscopes and cystoscopes with laser fiber could make a reduction in the risk of urethral damage in children. ${ }^{13}$

However, Ho:YAG bladder lithotripsy took a longer operative time than other choices principally for larger and multiple stones, this might increase the risk of urethral stricture subsequent to the urethral trauma in younger children due to smaller caliber of urethra. ${ }^{18}$

Ramakrishnan et al treated bladder stone in 23 children between 2-12 years (mean, 7.8 years) by Ho:YAG lithotripsy via 8 Fr ureteroscope. The MSS was $27 \mathrm{~mm}$ (range from 9 to $40 \mathrm{~mm}$ ) and they applied 0.6-1.8 $\mathrm{J}$ in a frequency of 5-12 Hz using 550 microns end-firing fiber. The MOT was 38 minutes (range, 19-62) and an 8 Fr urinary catheter was fixed for one night in patients. The mean hospital admission was 2.2 days also no residual stones or sever complications was reported. In mean follow-up of 42 months, none of the children developed urethral strictures. They mentioned that Ho:YAG cystolithotripsy could be an appropriate modality of treatment in children. ${ }^{19}$

As can be seen in Table 2, we compared the results of some studies about the different surgical managements of bladder stones in children with our results.

Although well-designed, this study has several limitations. First, this investigation was a retrospective study therefore we had some missing data especially in patients' follow up. Second, the sample size variation between the study groups was the other limitation of our study as well.

\section{Conclusion}

Our study revealed that Ho:YAG cystolithotripsy has a high success rate, is a minimally invasive management and is a safe method for bladder calculi without any need for stone extraction in children. Furthermore, shorter hospital stay, lack of scar, and not developing any complications (if proper equipment is available and done by expert hands) are the other advantages of this treatment.

\section{Ethical Considerations}

Researchers undertook to do the research subjects based on the ethical committee of Shahid Beheshti University of Medical Sciences.

\section{Conflict of Interests}

The authors declare no conflict of interest.

\section{Acknowledgments}

The authors wish to acknowledge the assistance of Urology staff.

\section{References}

1. Yoshida O. A chronological and geographical study on urolithiasis in Japan. Jpn J Endourol ESWL. 1990;3:5-10.

2. Schwartz BF, Stoller ML. The vesical calculus. Urol Clin North Am. 2000;27(2):333-346.

3. Trinchieri A. Epidemiology of urolithiasis: an update. Clin Cases Miner Bone Metab. 2008;5(2):101-106.

4. Abarchi H, Hachem A, Erraji M, Belkacem R, Outarahout N, Barahioui M. [Pediatric vesical lithiasis. 70 case reports]. Ann Urol (Paris). 2003;37(3):117-119.

5. Menon M. Urinary lithiasis: etiology, diagnosis, and medical management. In: Walsh PC, ed. Campbell's Urology. Saunder; 2002: 3229-3305.

6. Torricelli FC, Mazzucchi E, Danilovic A, Coelho RF, Srougi M. Surgical management of bladder stones: literature review. Rev Col Bras Cir. 2013;40(3):227-233. 
7. Ahmadnia H, Younesi Rostami M, Yarmohammadi AA, Parizadeh SM, Esmaeili M, Movarekh M. Percutaneous treatment of bladder calculi in children: 5 years experience. Urol J. 2006;3(1):20-22.

8. Razzaghi MR, Fallah Karkan M, Ghiasy S, Javanmard B. Laser Application in Iran Urology: A Narrative Review. $J$ Lasers Med Sci. 2018;9(1):1-6. doi:10.15171/jlms.2018.01

9. Fallah Karkan M, Ghiasy S, Ranjbar A, Javanmard B. Evaluation of $200 \mathrm{Mm}, 365 \mathrm{Mm}$ and $500 \mathrm{Mm}$ fibers of Ho:YAG laser in transurethral lithotripsy of ureteral: a randomize control trial. J Lasers Med Sci. 2018;9(1):69-72. doi:10.15171/jlms.2018.14

10. Papatsoris AG, Varkarakis I, Dellis A, Deliveliotis C. Bladder lithiasis: from open surgery to lithotripsy. Urol Res. 2006;34(3):163-167. doi:10.1007/s00240-006-0045-5

11. Aboulela W, ElSheemy MS, Shoukry AI, et al. Transurethral Holmium Laser Cystolithotripsy in Children: Single Center Experience. J Endourol. 2015;29(6):661-665. doi:10.1089/ end.2014.0636

12. Lal B, Paryani JP, Memon SU. Childhood bladder stones-an endemic disease of developing countries. J Ayub Med Coll Abbottabad. 2015;27(1):17-21.

13. Al-Marhoon MS, Sarhan OM, Awad BA, Helmy T, Ghali A, Dawaba MS. Comparison of endourological and open cystolithotomy in the management of bladder stones in children. J Urol. 2009;181(6):2684-2687. doi:10.1016/j. juro.2009.02.040

14. Teichman JM, Rogenes VJ, McIver BJ, Harris JM. Holmium:yttrium-aluminum-garnet laser cystolithotripsy of large bladder calculi. Urology. 1997;50(1):44-48. doi:10.1016/s0090-4295(97)00201-x
15. Grasso M. Experience with the holmium laser as an endoscopic lithotrite. Urology. 1996;48(2):199-206. doi:10.1016/s0090-4295(96)00158-6

16. Ahmadnia H, Kamalati A, Younesi M, Imani MM, Moradi M, Esmaeili M. Percutaneous treatment of bladder stones in children: 10 years experience, is blind access safe? Pediatr Surg Int. 2013;29(7):725-728. doi:10.1007/s00383013-3320-x

17. Isen K, Em S, Kilic V, Utku V, Bogatekin S, Ergin $\mathrm{H}$. Management of bladder stones with pneumatic lithotripsy using a ureteroscope in children. J Endourol. 2008;22(5):1037-1040. doi:10.1089/end.2007.0342

18. Wollin TA, Singal RK, Whelan T, Dicecco R, Razvi HA, Denstedt JD. Percutaneous suprapubic cystolithotripsy for treatment of large bladder calculi. J Endourol. 1999;13(10):739-744. doi:10.1089/end.1999.13.739

19. Ramakrishnan PA, Medhat M, Al-Bulushi YH, Gopakumar KP, Sampige VP, Al-Busaidy SS. Holmium laser cystolithotripsy in children: initial experience. Can J Urol. 2005;12(6):2880-2886.

20. Nameirakpam S, Naorem S, Faridi MS, Akoijam KS, Sinam RS. Transurethral Cystolithotripsy of Large Bladder Stones by Holmium Laser as a Day Care Procedure. J Clin Diagn Res. 2016;10(12):Pc01-pc02. doi:10.7860/ jcdr/2016/22493.8958

21. Salah MA, Holman E, Khan AM, Toth C. Percutaneous cystolithotomy for pediatric endemic bladder stone: experience with 155 cases from 2 developing countries. $J$ Pediatr Surg. 2005;40(10):1628-1631. doi:10.1016/j. jpedsurg.2005.06.039 